\title{
Partial Linear Gaussian Models for Tracking in Image Sequences Using Sequential Monte Carlo Methods
}

Elise Arnaud • Etienne Mémin

Published online: 8 July 2008

(C) Springer Science+Business Media, LLC 2008

\section{Erratum to: Int J Comput Vis (2007) 74: 75-102}

DOI 10.1007/s11263-006-0003-2

We would like to correct the following typo in our paper.

As shown in Fig. 1 and Fig. 6 and as correctly written in (Arnaud et al. 2005), the norm notations used in equations (20), (50) and (74) for the validation gate expressions have to be obviously replaced by an outer product. Equation (20) corrected reads:

$$
\begin{aligned}
\Sigma_{k}^{v g}= & \operatorname{var}\left[\mathbf{z}_{k} \mid \mathbf{z}_{1: k-1}\right] \\
\simeq & \sum_{i=1}^{N} w_{k-1}^{(i)}\left[R_{k \mid \mathbf{I}}+H_{k \mid \mathbf{I}} Q_{k \mid \mathbf{I}}^{(i)} H_{k \mid \mathbf{I}}^{t}+\mathbf{b}_{k \mid \mathbf{I}}^{(i)} \mathbf{b}_{k \mid \mathbf{I}}^{(i) t}\right] \\
& -\left(\sum_{i=1}^{N} w_{k-1}^{(i)} \mathbf{b}_{k \mid \mathbf{I}}^{(i)}\right)\left(\sum_{i=1}^{N} w_{k-1}^{(i)} \mathbf{b}_{k \mid \mathbf{I}}^{(i)}\right)^{t},
\end{aligned}
$$

The online version of the original article can be found under http://dx.doi.org/10.1007/s11263-006-0003-2.

E. Arnaud $(\bowtie)$

INRIA Rhône-Alpes/Universite Joseph Fourier, 38330

Montbonnot, France

e-mail: elarnaud@inrialpes.fr

E. Mémin

Universite Rennes I, Campus de Beaulieu, 35042 Rennes Cedex,

France

e-mail: memin@irisa.fr where $\mathbf{b}_{k \mid \mathbf{I}}^{(i)}=H_{k \mid \mathbf{I}} f_{k \mid \mathbf{I}}\left(\mathbf{x}_{k-1}^{(i)}\right)$. This slip of the pen has been pointed out by Prof. Xiaojun Wu, Jiangnan University, Wuxi, China. We thank him for bringing this to our attention.

\section{References}

Arnaud, E., Mémin, E., \& Cernuschi-Frias, B. (2005). Conditional filters for image sequence based tracking-Application to point tracking. IEEE Transactions on Image Processing, 14(1), 63-79. 